\title{
Research on fatigue life prediction of gear box based on collaborative simulation
}

\author{
Xiuju Du ${ }^{1, a}$, Yanhua Zhang ${ }^{1}$ and Changzhi $\mathrm{Jia}^{2}$ \\ ${ }^{1}$ Hebei Normal University, Shi Jiazhuang, Hebei, China \\ ${ }^{2}$ Ordnance Engineering College, China
}

\begin{abstract}
Because the static strength theory can't reflect exactly the dynamic loads suffered by gear box, it is difficult to analysis the gear box's reliability and rest life prediction with the short time and the complex task profile. In the paper, collaborative simulation based on interfaces is used in gear box. With the running simulation of different task profile the reliability and life prediction properly of all ingredients are analyzed under the dynamic loads, and with an example of planetary frame the feasibility is proved. The research on collaborative simulation based on interfaces used in gear box of tracked vehicle not only solve the technical problem which caused by the complex task profile but also join the engineer tightly, it is significant in practice.

Keywords: gear box, fatigue life prediction, collaborative simulation.
\end{abstract}

\section{Introduction}

The static strength theory can't reflect the dynamic loads practical in different working station of tracked vehicle which is designed with the static strength theory. So it results in the sharp difference of designed life and true lifetime, and in some degree it affect the reliability of whole system [1,2,3,4]. As the development of computer, multi-body dynamics and virtual prototyping, research on simulation, reliability and life prediction of complex tracked vehicle is feasible [5,6,7,8].

So collaborative simulation based on interfaces will solve not only the life prediction under dynamic loads but also contact tightly the engineering application. The results will be the important data to design and apply.

\section{Collaborative simulation based on interface}

\subsection{Loads analyzing of gear box}

Because of lacking of loads data before 1990's, the designed method of tracked vehicle still adopted traditional static strength theory. Depend on investigation of cars, more than 70 percent components are broken under dynamic loads. Compare tracked vehicle with car, the working condition is formidable natural conditions, so the proportion of broken components of tracked vehicle under dynamic loads is more than cars'. So the dynamic loads under different task station are basic and important to tracked vehicle gear box's design. While dynamic loads are influenced by many motors such as working

a Corresponding author: dxj_hbsd@126.com 
conditions, structural parameter and road surface. So the research on dynamic loads is difficult because of not only long time and huge cost but also the acquisition model. Nowadays experiment is considered the effective method to achieve dynamic loads, while the test method is limit due to the little versatility of experiment results.

\subsection{Collaborative simulation based on interfaces used in tracked vehicle gear box}

In this paper collaborative simulation based on interfaces is researched on tracked vehicle which involved in multi-disciplinary crossing and merging. That combined simulation with engineer application. Meanwhile collaborative simulation based on interfaces can implement some experiments unable to realize. For example, running simulation can instead in some degree the real experiment properly. Combine the results from the running simulation with dynamic simulation, FEA analysis, fatigue simulation and so on will enlarge the area of simulation. Collaborative simulation based on interfaces tightly combined with engineering will increase reliability of whole system and generate huge economic benefit. The flow chart of collaborative simulation used in tracked vehicle gear box is provided in Fig.1 [4].

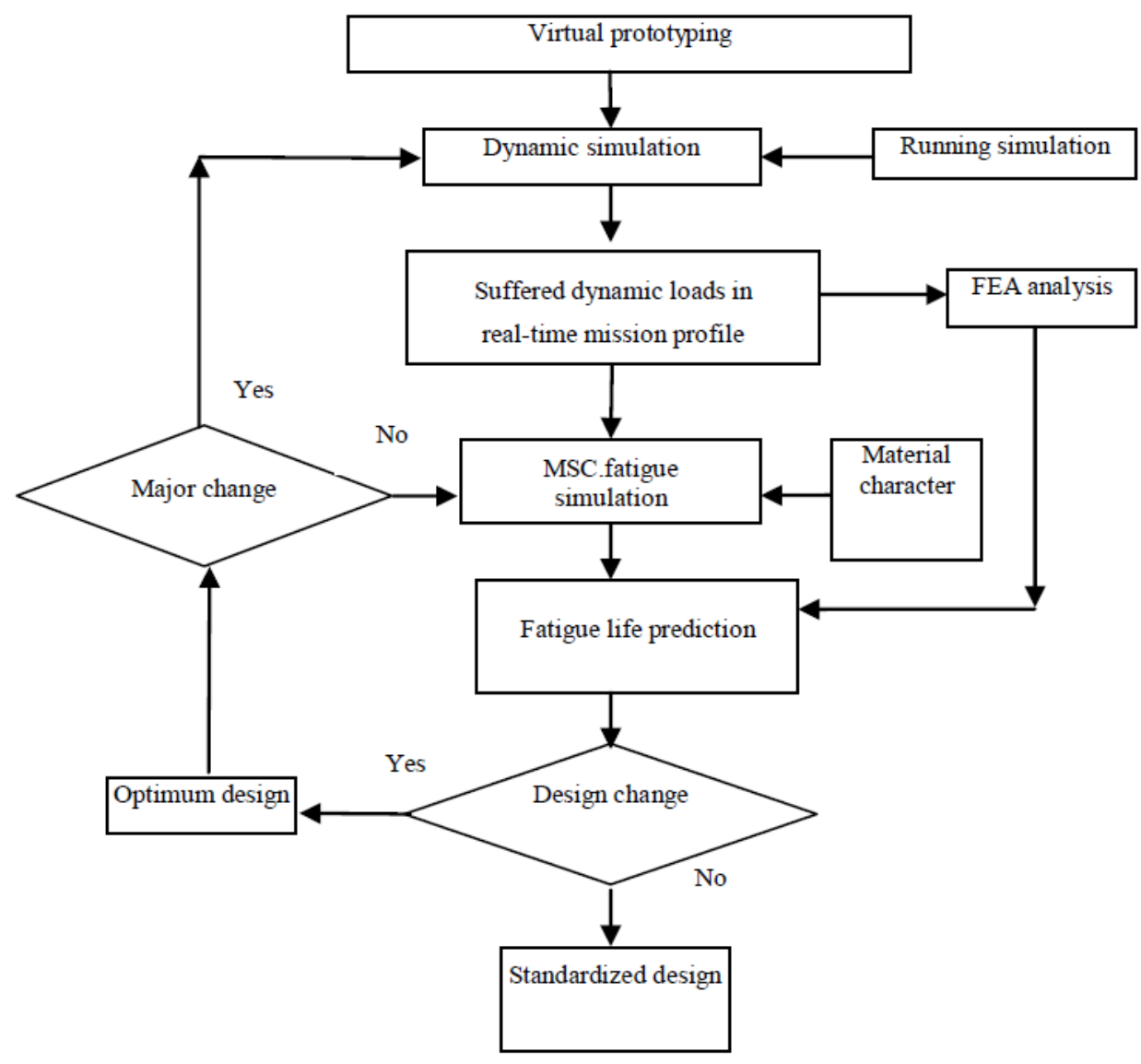

Figure 1. The flow chart of collaborative simulation based on interfaces applied in tracked vehicle gear box.

With the flow chart the dynamic loads suffered in different working condition achieved with running simulation. The dynamic loads are important data to dynamic simulation and FEA analysis. Based on the results fatigue life prediction and optimum design are researched [3]. 


\section{Case study}

The case study put the fig. 1 into practice which increases the life of the planetary frame in gear box, and combined ADAMS, MSC. Patran and FE-FATIGUE with collaborative simulation are based on interfaces. In the paper the virtual prototyping of tracked vehicle gear box is built up. Combined with the running simulation of tracked vehicle gear box, the loads of every part suffered will be obtained [2]. As we all know that the dynamic loads are the key to the structural reliability in the collaborative simulation. In the paper the planetary frame in gear box certificate the feasibility of collaborative simulation based on interfaces applied in tracked vehicle gear box. Virtual prototyping of tracked vehicle gear box is list in Fig.2.

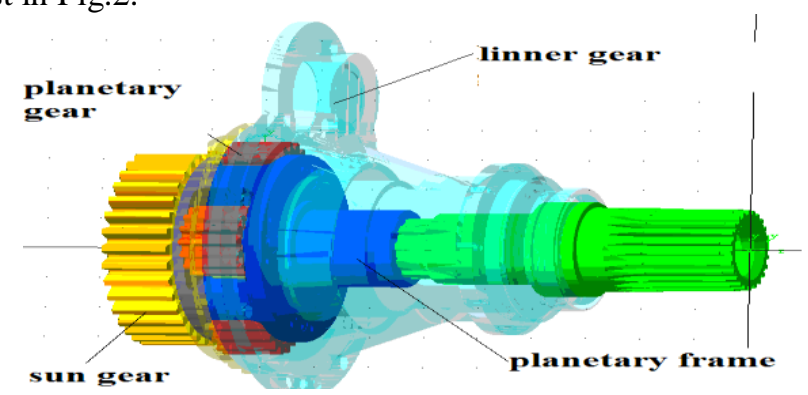

Figure 2. Virtual prototyping of tracked vehicle gear box.

With the virtual prototyping of tracked vehicle gear box the running simulation proceeded on different roads with MSC.ATV, the dynamic loads suffered by the planetary frame in one task profile is obtained as Fig.3.

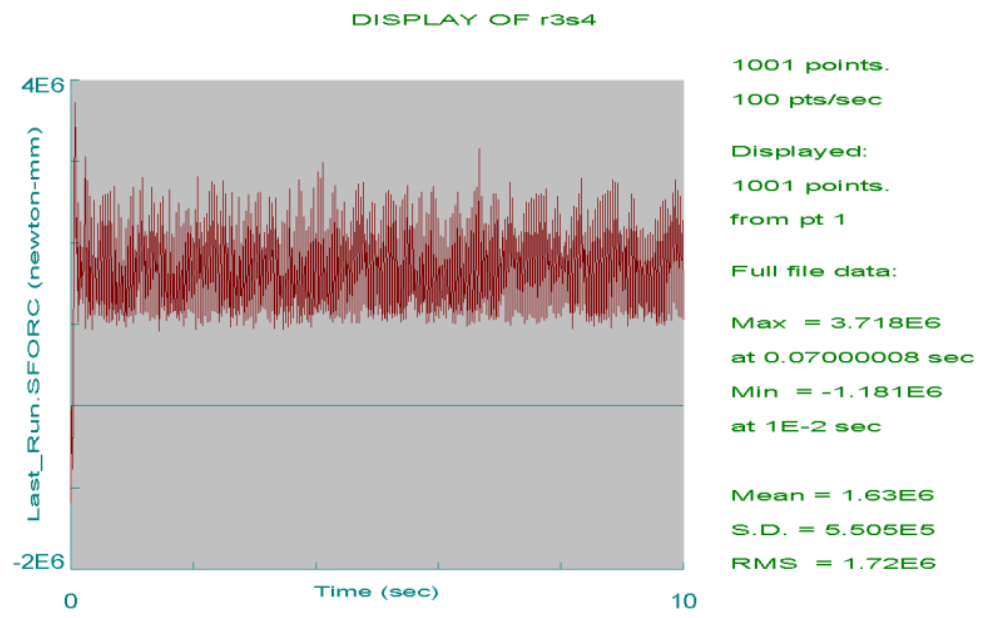

Figure 3. The dynamic loads suffered by the planetary frame.

Under the effect of dynamic loads showed on fig.3, the finite element analysis and fatigue analysis supply the fatigue life contours of the planetary frame as fig.4. In fact, the planetary frame is a main part and easy to breakdown, so it is necessary to redesign to increase the reliability. The life of initial structural is shown in fig.4. Because the planetary gears are important sector in the life prediction, so in the paper with the permission of drive ratio the planetary gears are changed from four to six. Collaborative simulation based on interfaces applied in the structural optimism again, so the life of the planetary frame with the structural optimism is shown in fig.5 [1]. 


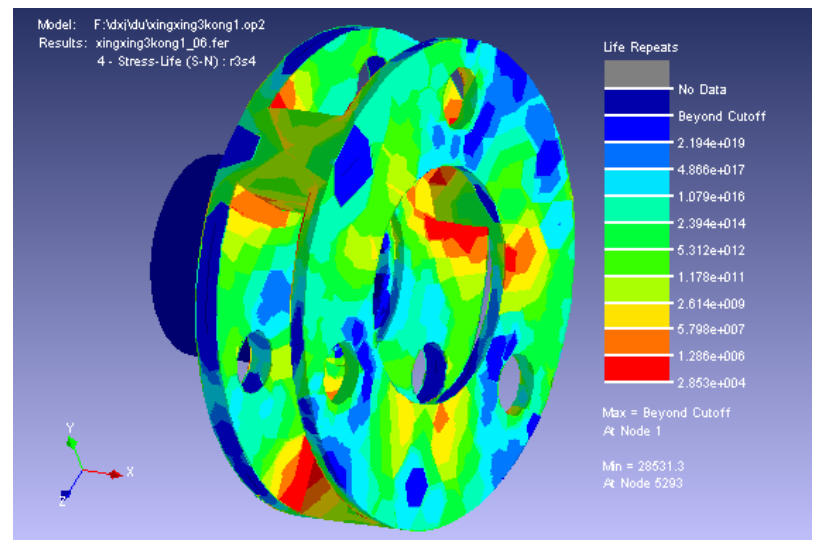

Figure 4. Fatigue life of the planetary frame.

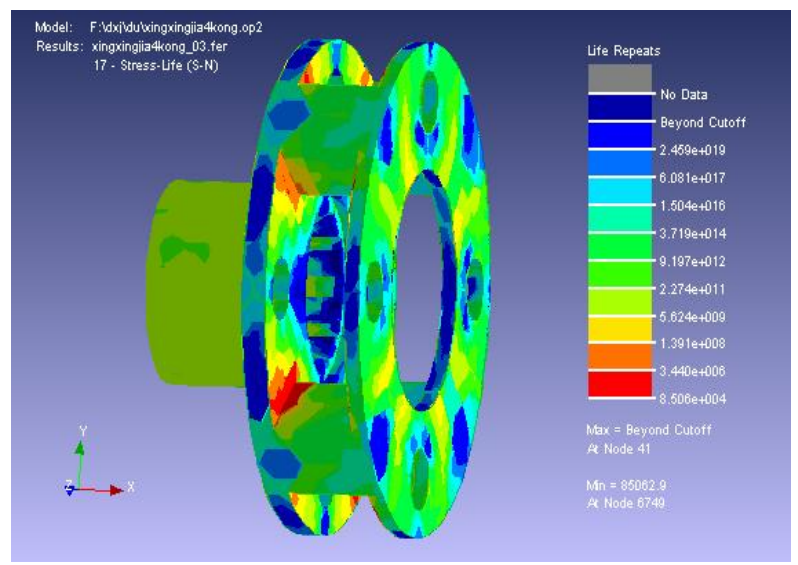

Figure 5. Fatigue life of the redesign planetary frame.

The results of the comparison showed that the redesign construction's fatigue life is much longer than before. So the redesign planetary frame not only solved the faults basically, but also increased the reliability of tracked vehicle gear box significantly. What's more, the example proved the feasibility of collaborative simulation based on interfaces applied in tracked vehicle gear box and will generate huge economic impact on developing weapon.

\section{Summary}

This paper researched collaborative simulation based on interfaces applied in tracked vehicle gear box. The research solves not only the problem of life prediction and structural optimism existed under dynamic loads in practice but also combine the engineer application tightly. With a example show that collaborative simulation based on interfaces applied in planetary frame's life prediction and structural optimism is reasonable well.

\section{Acknowledgements}

The paper is sponsors by the Science and Technology Project of Hebei Provine (item number:16211806D and 15210701D), and same time sponsors by the Key Projects of Hebei Education Department(item number:ZD2016084). 


\section{References}

1. BI Lu-yan, JIAO Zong-xia,FAN Sheng-tao, "Comprehensive Electromechanical Systems Multi-disciplinary Design and Simulation Integrated Framework", Journal of System Simulation, vol.20(12):pp.3265-3269, 2008.

2. James E.Crosheck, "The integration of analysis and text for full vehicle structural durability", NDIA SBA Conference, May 15-17, 2001.

3. Xiong Guangleng, Guo Bin \& Chen Xiaobo. Collaborative simualation \& virtual propotyping[M],TSINGHUA UNIVERSITY PRESS, 2004.

4. D. Rubinstein, R. Hitron, A detailed multi-body model for dynamic simulation of off-road tracked vehicles, Journal of Terramechanics 41 (2004) 163-173.

5. DU Xiuju; DONG Zhaowei \& WANG Xinggui..Research on collaborative simulation based on interfaces used in weapon system. JOURNAL OF SYSTEM SIMULATION, 2006; 18(5):1 371-1 374.

6. Gianni F, Gian A M, Paolo R. Virtual Prototyping of Mechatronic System [J].Annual Review in Control, 28: 193 206, 2004.

7. Zheng Hong-tao,Cai Zhi-hao,Peng Xiao-yuan,'Research of Virtual Prototype MDO Problem Modeling".Journal of System Simulation, vol.37(10), pp.2749 2752, 2006.

8. Du Xiuju, Jia Changzhi, "Application of Collaborative Simulation Based on Interfaces Used in Dynamic Optimization Design”. Chinese Journal of Mechanical Enegineering, vol.44(8), pp123-131, 2008. 